\title{
Effect of single string structure and multiple string interaction on strange particle production in $p p$ collisions at $\sqrt{s}=7 \mathrm{TeV}$
}

\author{
Liang Zheng,,${ }^{1,2,{ }^{*}}$ Dai-Mei Zhou, ${ }^{2, \dagger}$ Zhong-Bao Yin, ${ }^{2, \ddagger}$ Yu-Liang Yan,,${ }^{3,2}$ Gang Chen, ${ }^{1}$ Xu Cai, ${ }^{2}$ and Ben-Hao Sa ${ }^{3,2, \S}$ \\ ${ }^{1}$ School of Mathematics and Physics, China University of Geosciences (Wuhan), Wuhan 430074, China \\ ${ }^{2}$ Key Laboratory of Quark and Lepton Physics (MOE) and Institute of Particle Physics, Central China Normal University, \\ Wuhan 430079, China \\ ${ }^{3}$ China Institute of Atomic Energy, P.O. Box 275 (18), Beijing 102413, China
}

(Received 6 July 2018; published 25 September 2018)

\begin{abstract}
We present a systematic study of the strange particle production at the CERN Large Hadron Collider (LHC) in proton-proton $(p p)$ collisions at $\sqrt{s}=7 \mathrm{TeV}$ based on PACIAE simulations. Two different mechanisms accounting for single string structure variations and multiple string interactions are implemented in the simulations. These modifications give rise to increased effective string tension in the Lund fragmentation model and generate more strange particles in the hadronic final state. By comparing the results with a wealth of the LHC data, we find that the inclusion of variable effective string tension yields an improved agreement between theory and experiment, especially for the recently observed multiplicity dependence of strangeness enhancement in $p p$ collisions. This approach provides us a new method to understand the microscopic picture of the novel high multiplicity $p p$ events collected at the LHC in the string fragmentation framework.
\end{abstract}

DOI: 10.1103/PhysRevC.98.034917

\section{INTRODUCTION}

The production of strange hadrons plays an important role in investigations of properties of the strong force and deconfined quark-gluon matter. For hadronic scatterings in vacuum, the strangeness content in the created particles is much smaller compared to the nonstrange components due to the mass suppression effect in the particle production. If a quark-gluon plasma (QGP) is produced, the chemically equilibrated system generates a high number of strange quark pairs through the thermal gluon fusion process and also favors the formation of multistrange hadrons [1,2]. It is thus believed that the enhancement of strange particle production, especially for multistrange baryons, observed in heavy-ion collision experiments [3-5] is a characteristic signature for the presence of QGP matter [6].

Recently, striking commonalities were observed between high multiplicity $p p$, proton-lead ( $p-\mathrm{Pb})$, and heavy-ion collisions at energies available at the CERN Large Hadron Collider (LHC). The strangeness production, for example, has been systematically analyzed by the ALICE Collaboration with a

\footnotetext{
*zhengliang@cug.edu.cn

†'zhoudm@mail.ccnu.edu.cn

${ }^{\ddagger}$ zbyin@mail.ccnu.edu.cn

§sabh@ciae.ac.cn
}

Published by the American Physical Society under the terms of the Creative Commons Attribution 4.0 International license. Further distribution of this work must maintain attribution to the author $(s)$ and the published article's title, journal citation, and DOI. Funded by $S C O A P^{3}$. wide range of experimental data from $p p, p-\mathrm{Pb}$, and lead-lead $(\mathrm{Pb}-\mathrm{Pb})$ collisions [7]. A pronounced enhancement of strange particle relative to pion production is reported in Ref. [7]. The strangeness-to-pion ratio increases smoothly with the event multiplicity across all collision systems. The magnitude of enhancement relies on the strangeness content of the hadron. The particle ratios obtained in $p p$ are quite similar to those found in $p-\mathrm{Pb}$ at the same event multiplicities. These intriguing results complement other observations [8-12] showing similar features traditionally associated with the QGP formation, and they call for further theoretical investigations to understand the microscopic mechanisms that lead to these novel phenomena.

Different models are used to interpret this universal strangeness-to-pion ratio as a function of the event multiplicities. Statistical-thermal model calculations are found to provide a reasonable quantitative description to the particle ratios observed in data, and they suggest that the suppression of strange hadron production in $p p$ may come from the explicit conservation of strangeness quantum number based on the canonical approach [13]. The EPOS model [14] assumes the QGP matter is partly formed in $p p$ collisions, treating the interactions based on a core-corona approach. The prediction of this model agrees qualitatively with the observed increasing trend of the strangeness production [15]. Another model which qualitatively describes the data is the color ropes mechanism [16,17] implemented in DIPSY [18], taking into account the color interactions between strings. Other interesting extensions to the Lund string fragmentation model are implemented in PYTHIA8 [19] by considering thermodynamic features of strings in a dense environment [20], and are used to explain some of the changing flavor composition with multiplicity. Although various models can describe some key features 
of the data, the fundamental origin of enhanced strangeness production in $p p$ collisions is still largely unknown.

In this work, we introduce an effective string tension stemming from the single string structure variation and the multiple string interaction effect to reduce the strange quark suppression in $p p$ collisions based on the tunneling probability in the Lund string fragmentation model. These two mechanisms of obtaining the effective string tensions are implemented in the PACIAE Monte Carlo event generator [21] to study the strange hadron production in $\sqrt{s}=7 \mathrm{TeV} p p$ collisions at the LHC. Systematic studies are performed to compare the effects of different effective string tensions on the multiplicity dependence of strange flavor composition.

The remainder of the paper is organized as follows: in Sec. II, we give a short introduction to the approaches to construct the effective string tension in the string fragmentation model. Detailed implementations of the effective string tension mechanism in the PACIAE model are illustrated in Sec. III. The results and comparisons to data are provided in Sec. IV. We summarize in Sec. V.

\section{EFFECTIVE STRING TENSION}

In the Lund string fragmentation model, particles are produced mainly through the iterative breakups of the color singlet string pieces created during the multiple parton-parton interactions in a $p p$ collision [22]. Given an initial string object consisting of $q_{0}$ and $\overline{q_{0}}$ endpoints moving apart from each other, it is assumed that the original string system can break up into two, with the production of a new $q_{1} \overline{q_{1}}$ pair in the middle of two end quarks. Therefore, one can find a new hadron formed from the $q_{0} \overline{q_{1}}$ object, leaving $q_{1}$ behind, which may at a later stage pair with other iterative creations or with $\overline{q_{0}}$ directly. The hadron's information is then determined by the parton flavor and kinematic configurations of its quark components. If a diquark-antidiquark pair is generated at the breakup point instead of the $q \bar{q}$ pair, a baryon can be created in the same formalism. As required by the local flavor conservation, the new $q \bar{q}$ pair is always produced at a common vertex. Similar to the Schwinger particle production model in an electric field [23], virtual particles only hadronize when the $q \bar{q}$ pair tunnels out a distance $d=m_{\perp} / \kappa$. The tunneling probability can thus be obtained as

$$
\begin{aligned}
P\left(m_{\perp q}\right) & \propto \exp \left(-\frac{\pi}{\kappa} m_{\perp q}^{2}\right) \\
& =\exp \left(-\frac{\pi}{\kappa} m_{q}^{2}\right) \exp \left(-\frac{\pi}{\kappa} p_{\perp q}^{2}\right),
\end{aligned}
$$

where $\kappa$ is the string tension representing the color force acting on the quarks in the linear confinement field.

This formula suggests the relative production of different quark flavors depends on the effective quark mass involved in the tunneling probability. As it is practically hard to give the mass values theoretically, the relative production probabilities are usually treated as empirical model parameters tuned to data. The string tension value is often assumed to be $\kappa \approx 1 \mathrm{GeV} / \mathrm{fm}[24,25]$ for a pure $q \bar{q}$ dipole string hadronized without interactions to its close-by neighbors. However, for the strings created in $p p$ collisions at LHC energies, the structure of a string can be much more complicated than the pure dipole state. and the hadronization of each string may not be treated independently when the interaction between neighboring strings becomes non-negligible. To quantitatively account for these impacts, one can apply an effective string tension in the tunneling probability, Eq. (1), for the estimation of flavor compositions.

In this work, the effects of gluon wrinkling in the string structure and the multiple string interactions in the densely populated environment are included in the effective string tensions, individually and taken together. The first scheme follows the reduction of the strange quark suppression mechanism [26] modeled in PACIAE [21] which enhances the string tension when radiated gluons exist on a string piece. The parametrized effective string tension responsible for the single string structure change can be given as

$$
\kappa_{\mathrm{eff}}^{s}=\kappa_{0}(1-\xi)^{-\alpha},
$$

where $\kappa_{0}$ is the pure $q \bar{q}$ string tension usually set to $1 \mathrm{GeV} / \mathrm{fm}$, $\alpha$ is a parameter to be tuned with experimental data, while $\xi$ can be parametrized as

$$
\xi=\frac{\ln \left(\frac{k_{\perp \max }^{2}}{s_{0}}\right)}{\ln \left(\frac{s}{s_{0}}\right)+\sum_{j=\text { gluon }} \ln \left(\frac{k_{\perp j}^{2}}{s_{0}}\right)},
$$

with $k_{\perp}$ being the transverse momentum of the gluons inside a dipole string. $\sqrt{s}$ and $\sqrt{s_{0}}$ give the mass of the string system and a parameter related to the typical hadron mass, respectively. $\xi$ quantifies the difference between a gluon wrinkled string and a pure $q \bar{q}$ string. The fractal structure of a string object is dominated by the hardest gluon on the string. The quantity $\xi$ is defined to measure the fraction of the multiplicity introduced by the hardest gluon in a string object. $(1-\xi)^{-1}$ in Eq. (2) thus describes the multiplicity enhancement factor of the hardest gluon compared to the rest of the string component, and can be related to the string tension with a scaling formula. The value of this string tension changes on a string-by-string basis in the current implementation and takes the string-wise fluctuations into consideration.

On the other hand, we consider the multiple string interaction effects from the correlation of strings overlapping in a limited transverse space by parametrizing the effective string tension, in a manner similar to the close-packing of strings discussed in Ref. [20], as follows:

$$
\kappa_{\mathrm{eff}}^{m}=\kappa_{0}\left(1+\frac{n_{M P I}-1}{1+p_{T \text { ref }}^{2} / p_{0}^{2}}\right)^{r},
$$

in which $n_{M P I}$ indicates the number of multiple parton interactions in a $p p$ collision event and $p_{T \text { ref }}^{2} / p_{0}^{2}$ shows the transverse scale of a typical string object relative to the proton size. The exponent $r$ is then treated as a free parameter. Again, $\kappa_{0}$ provides the string tension without any modifications and takes the same value as used in Eq. (2). As larger $n_{M P I}$ leads to a denser string system in an event, $n_{M P I}$ is strongly correlated with the number of charged particles. Together with the typical string capacity factor $p_{T}^{\text {ref }} / p_{0}$ of a proton target in the transverse space, Eq. (4) then characterizes the 
overlap degree of string objects created in one event. Unlike the close-packing effective string tension in Ref. [20], $\kappa_{\text {eff }}^{m}$ is supposed to capture the overall event activity feature, and provides a generic way to model the interstring interaction effect without considering the differential picture of the string population. This multiple string interaction triggered effective tension applies globally to all the string objects in the same event, and thus serves as an event-wise effective string tension moderator. It is expected that the effect of interstring interactions can be modeled in this functional form without considering the details of individual string dynamics.

Additionally, the string-wise single string structure change and event-wise multiple string interaction effect can be combined together by replacing the $\kappa_{0}$ in Eq. (4) with $\kappa_{\text {eff }}^{s}$. Assuming $\kappa_{0}=1 \mathrm{GeV} / \mathrm{fm}$, one can derive a combined effective string tension as a product of the two:

$$
\begin{aligned}
\kappa_{\mathrm{eff}}^{s+m} & =\kappa_{\mathrm{eff}}^{s}\left(1+\frac{n_{M P I}-1}{1+p_{T \text { ref }}^{2} / p_{0}^{2}}\right)^{r} \\
& =\kappa_{\mathrm{eff}}^{s} \times \kappa_{\mathrm{eff}}^{m} .
\end{aligned}
$$

By introducing the effective string tension, the quark relative production ratio parameters can be modified by a scaling relation, as implied in the tunneling probability.

\section{THE SIMULATION FRAMEWORK}

The variational string tension study was performed in this work with PACIAE simulations. The PACIAE model is a multipurpose Monte Carlo event generator developed to describe a wide range of collisions, including hadron-hadron interactions, hadron interactions off nuclei, and nucleus-nucleus collisions. It is built based on PYTHIA-6.4 [27] and incorporates parton and hadron rescattering stages to take care of the nuclear medium effects. For $p p$ collisions, the PACIAE model is different from PYTHIA, with the capability of simulating the parton cascade before hadronization and hadron rescattering after hadronization. We follow the strategy in Refs. [21] and [18], which introduce a modification of the relevant string fragmentation parameters to account for the string tension alterations. The following relevant string fragmentation parameters are supposed to evolve with the string tension directly:

(1) $\rho$, strange to light quark ratio $P(s) / P(u), \operatorname{PARJ}(2)$ in PYTHIA;

(2) $x$, extra suppression on diquarks with strange content, PARJ(3) in PYTHIA;

(3) $y$, spin 1 to spin 0 diquark ratio, PARJ(4) in PYTHIA;

(4) $\sigma$, Gaussian width of the transverse momentum distribution for primary hadrons in fragmentation, PARJ(21) in PYTHIA.

The overall diquark-to-quark ratio $P(q q) / P(q), \quad \eta$ [PARJ(1) in PYTHIA], relies on the above parameters and changes with the string tension indirectly. We take the pretuned parameters based on the inclusive measurements as the reference values at $\kappa=\kappa_{0}$ and modify these parameters according to a simple scaling indicated by Eq. (1). This means if $\kappa=\kappa_{\text {eff }}$, then $\rho_{\text {eff }}=\rho_{0}^{\kappa_{0} / \kappa_{\text {eff }}}, x_{\text {eff }}=x_{0}^{\kappa_{0} / \kappa_{\text {eff }}}, y_{\text {eff }}=y_{0}^{\kappa_{0} / \kappa_{\text {eff }}}$,

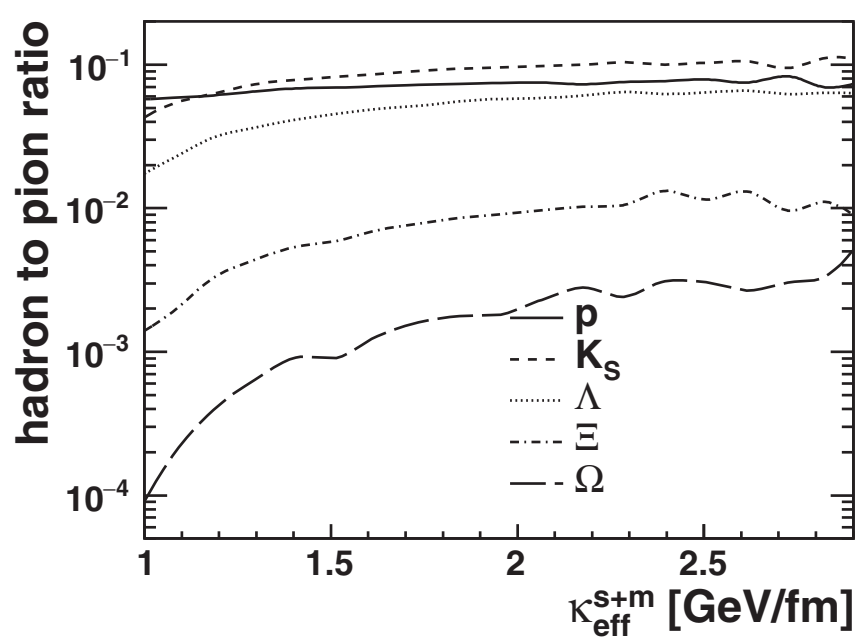

FIG. 1. Relative production with respect to $\pi$ for different particles in the combined string tension change scheme, varying with $\kappa_{\mathrm{eff}}^{s+m}$ in $\sqrt{s}=7 \mathrm{TeV} p p$ collisions.

and $\sigma_{\text {eff }}=\sigma_{0} \sqrt{\kappa_{\text {eff }} / \kappa_{0}}$. However, the diquark-to-quark ratio needs to be revised to $\eta_{\text {eff }}=w_{\text {eff }} \beta\left(\frac{\eta_{0}}{w_{0} \beta}\right)^{\kappa_{0} / \kappa_{\text {eff }}}$, where $w_{\text {eff }}=$ $\frac{1+2 x \rho+9 y+6 x y \rho+3 x^{2} y \rho^{2}}{2+\rho}$ depicts the weighting factor from all different kinds of diquark or quark combinations. As diquarks in the model are effectively generated through a stepwise popcorn mechanism [28] from the successive production of several $q \bar{q}$ pairs, $\beta$ is introduced for the probability to have a $q \bar{q}$ fluctuation which is primarily independent of the string tension.

The reference values for the above pretuned string fragmentation parameters are taken from the "Perugia 2011" tune [29] [PARJ $(1)=0.087, \operatorname{PARJ}(2)=0.19, \operatorname{PARJ}(3)=$ $0.95, \operatorname{PARJ}(4)=0.043, \operatorname{PARJ}(21)=0.33$ ], which provides a reasonable description of the inclusive measurements at LHC energy. To describe the charged particle density, a factor $K=0.82$ needs to be introduced in PACIAE for the hard scattering cross sections. The fraction of diquarks from twostep quark-antiquark pair productions, $\beta$, is found to be important for the multiplicity dependence of the baryon-tomeson ratio. We fix $\beta=0.05$ to give flat $\Lambda / K_{S}^{0}$ and $p / \pi$ ratios dependent on event multiplicity. The single string structure effective string tension $\kappa_{\text {eff }}^{s}$ and related parameters $\alpha=3.5$ and $s_{0}=0.8 \mathrm{GeV}$ are taken from an earlier fit to experimental data in Ref. [26]. For the multiple string interactions, we set $p_{T \text { ref }}^{2} / p_{0}^{2}=1$ and $r=0.2$ in this work.

Figure 1 shows different particleto- $\pi$ ratios varying with the string tension based on the parameter setup discussed above for $p p$ collisions at $\sqrt{s}=7 \mathrm{TeV}$. The result is given by the combined effective string tension $\kappa_{\text {eff }}^{s+m}$ simulation with both string structure change and multiple string interactions shown in Eq. (5). We find a rapid increase with $\kappa_{\text {eff }}^{s+m}$ in strange particle relative production, especially for the multistrange particles. Due to the choice of a small $\beta$ value, the $p / \pi$ ratio barely changes with respect to the string tension.

We also explore the string tension varying with the event multiplicity in different scenarios, as shown in Fig. 2. The event-wise track numbers at forward rapidities are used as 

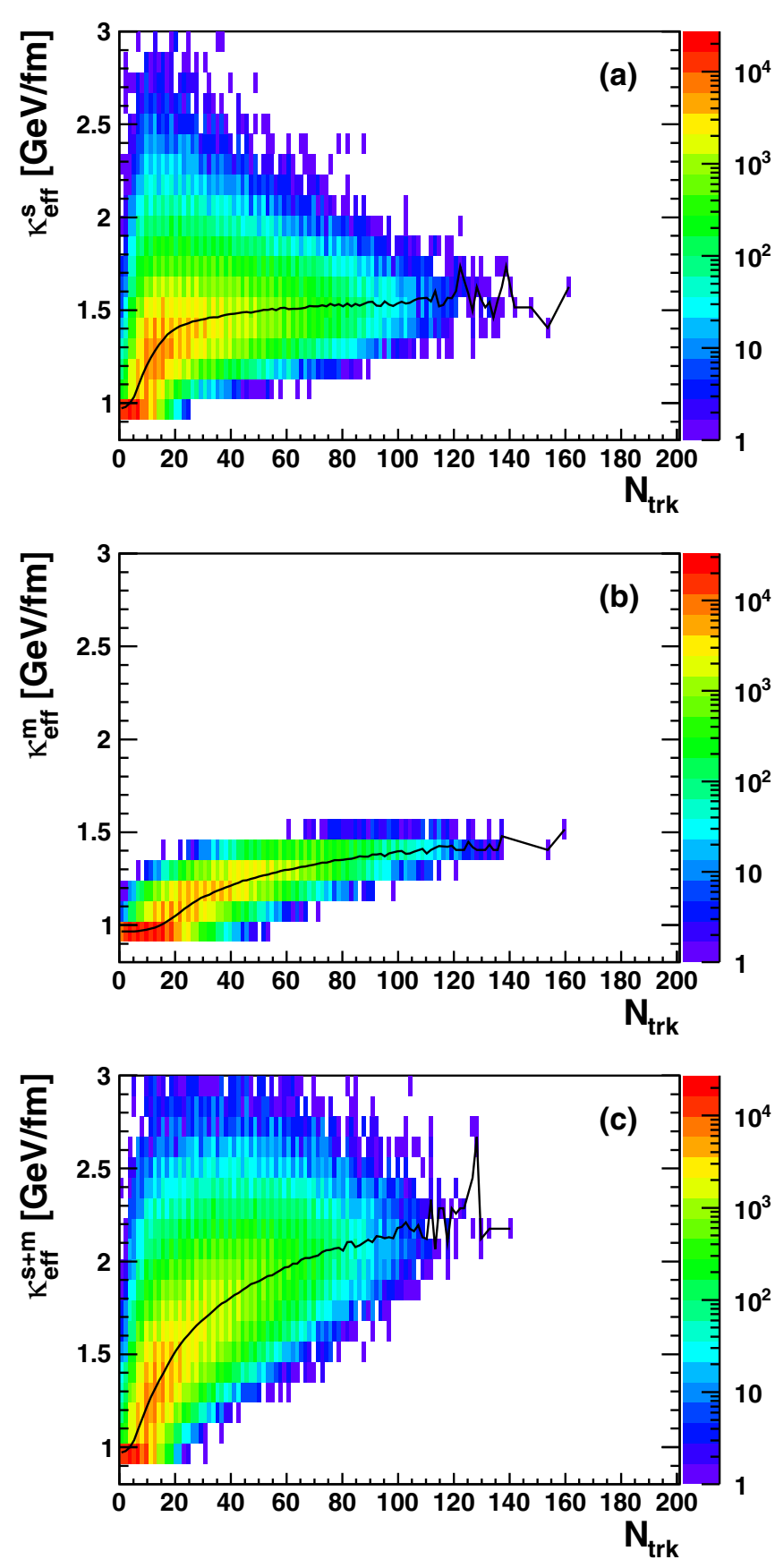

FIG. 2. Correlation of the effective string tension and the number of charged tracks accepted within the regions $-3.7<\eta<-1.7$ and $2.8<\eta<5.1$ (following the ALICE analysis convention) in $p p$ collisions at $\sqrt{s}=7 \mathrm{TeV}$ for single string structure change $\kappa_{\text {eff }}^{s}$ (a), multiple string interaction effect $\kappa_{\text {eff }}^{m}$ (b), and combined string tension $\kappa_{\text {eff }}^{s+m}(\mathrm{c})$. The solid line shows the average value of the effective string tension, dependent on multiplicity, in each scenario.

the event activity estimator. In this comparison, one can observe that there is a correlation between the effective string tension and the event multiplicity. For the single string structure related effective string tension $\kappa_{\text {eff }}^{s}$, a rapid increase of string tension is expected in the low multiplicity region due to stronger gluon radiation. The maximum gluon radiation energy is constrained by the allowed phase space. For the events with high multiplicities, the effects from the single string structure change may not be important anymore. Accordingly, if we switch to the multiple string interaction case, the average string tension $\kappa_{\mathrm{eff}}^{m}$ of an event is rising with the multiplicity, with rather small variations. By combining the effects of the two modifications in the effective string tension $\kappa_{\text {eff }}^{s+m}$, we get an even larger average tension, as the coupling of two scenarios will amplify this effect.

\section{RESULTS}

To investigate the effects of the different effective string tension scenarios, we focus on $p p$ collisions with $\sqrt{s}=7 \mathrm{TeV}$. The inclusive measurements of the charged particle pseudorapidity, transverse momentum, and multiplicity distributions are presented in Fig. 3. Model predictions with default string tension $\kappa_{0}$, single string structure change $\kappa_{\text {eff }}^{s}$, and multiple string interactions $\kappa_{\text {eff }}^{m}$ are shown with the solid, dashed, and dotted lines, respectively. The combination of two varying string tension scenarios $\kappa_{\mathrm{eff}}^{s+m}$ is shown with the dashdotted line. It is shown in this comparison that the charged density decreases as the effective string tension increases. This is not beyond expectations in the model, as increasing string tension makes it more difficult for a particle to tunnel out. This effect can also be identified in the mid-rapidity event multiplicity distribution, where high multiplicity events become rare in $\kappa_{\text {eff }}^{s}$ and $\kappa_{\text {eff }}^{s+m}$ simulations, as shown in Fig. 3(c). However, the impact on the charged particle transverse momentum distribution is hardly visible with the inclusion of different string tension parametrizations indicated by Fig. 3(b).

The multiplicity dependent studies are made with the event classifier counting the number of charged particles accepted in the pseudo-rapidity regions $-3.7<\eta<-1.7$ and $2.8<$ $\eta<5.1$, following the ALICE data analysis method. Based on the multiplicity distribution of charged particles in the forward rapidity region, the same event sample is divided into ten event classes with the same percentile definition as in Ref. [7]. For each event class, one can estimate the charged particle pseudopraidity density within $|\eta|<0.5$ to represent the event activity and study the per-rapidity strange particle production in $|y|<0.5$. The average charged densities in each event class for different string tension implementations are presented in Table I. It can be seen that the average charged densities in each event class are close to the measured data for variations of effective string tensions. In event class I, the average charged density with large effective string tensions $\kappa_{\text {eff }}^{s+m}$ becomes about $20 \%$ smaller than the case with a $\kappa_{0}$ string tension assumption. This is also consistent with our observation regarding the reduction of high multiplicity events, as shown in Fig. 3(c), with increasing string tension.

We perform an examination of the multiplicity dependence of charged pion and proton yields as shown in Fig. 4. The results suggest that the total pion production in each event class only slightly decreases with the increasing string tension. It is then not surprising to see that the $\pi$ yields from all four scenarios are in reasonable agreement with the experimental data. On the other hand, the integrated proton yield from the model is higher than the data, even with the constant string 

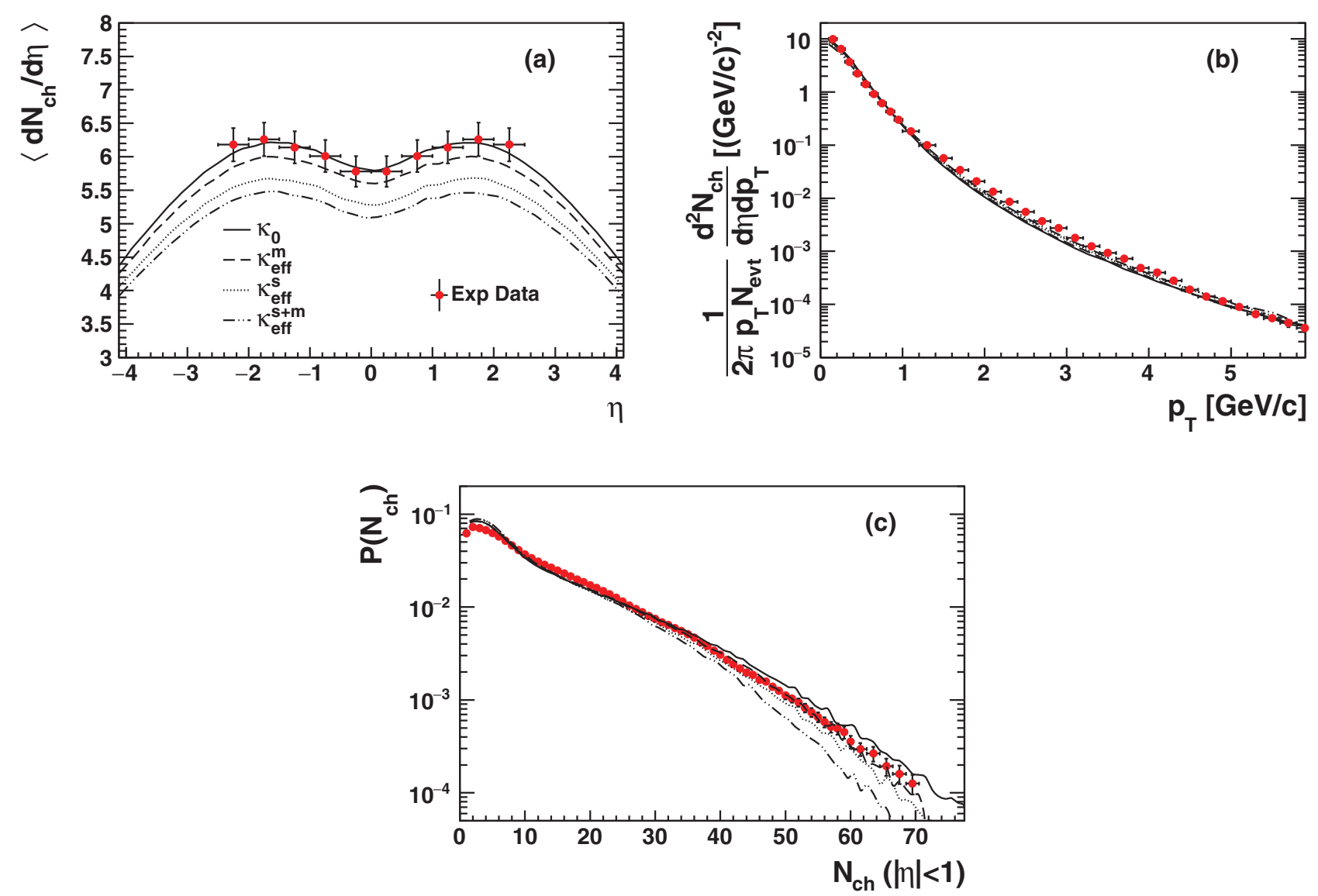

FIG. 3. Charged particle distributions shown in pseudorapidity (a), transverse momentum (b), and multiplicity (c) spaces for $p p$ collisions at $\sqrt{s}=7 \mathrm{TeV}$. Mid-rapidity charged particle numbers are obtained for $|y|<1$. Four different scenarios are shown for the $\kappa_{0}, \kappa_{\mathrm{eff}}^{s}, \kappa_{\mathrm{eff}}^{m}$, and $\kappa_{\text {eff }}^{s+m}$ string tension setups. The experimental data are taken from [30,31].

tension $\kappa_{0}$. All effective string tension variation scenarios introduce a negligible increase to the integrated proton yield, comparing with the constant string tension result. It our setup of the small $\beta$ parameter which determines how strongly the diquark-to-quark production ratio relies on the string tension.

In Fig. 5, we show a comparison of simulation results on the multiplicity dependence of strangeness production with experimental data. As can be seen in Fig. 5(a), $K_{S}^{0}$ production is enhanced with the inclusion of a stronger string tension. Comparing the $K_{S}^{0}$ production in the events with the largest charged particle density between the $\kappa_{0}$ and $\kappa_{\text {eff }}^{s+m}$ scenarios, the yield is increased by a factor of $50 \%$. The impact of the string tension change on strange baryon production is much more pronounced than that on $K_{S}^{0}$. In the strange baryon comparisons, the slope change due to string tension variation shows a clear hierarchy depending on the strangeness number. The difference between the slope of the minimum string tension case $\left(\kappa_{0}\right)$ and the maximum string tension case $\left(\kappa_{\mathrm{eff}}^{s+m}\right)$ in $\Lambda$ production is the smallest, while the $\Omega$ slope changes most dramatically from the $\kappa_{0}$ case to the $\kappa_{\text {eff }}^{s+m}$ case. This strangeness dependent slope change is a direct outcome, as our implementation involves the variation of the effective string tension, which determines the relative production of the

TABLE I. Charge density in each event class $\left\langle d N_{c h} / d \eta\right\rangle_{|\eta|<0.5}$. Data are taken from Ref. [7].

\begin{tabular}{|c|c|c|c|c|c|c|c|c|c|c|}
\hline & \multicolumn{10}{|c|}{ Event class } \\
\hline & I & II & III & IV & V & VI & VII & VIII & IX & $\mathrm{X}$ \\
\hline Expt. data & $21.3 \pm 0.6$ & $16.5 \pm 0.5$ & $13.5 \pm 0.4$ & $11.5 \pm 0.3$ & $10.1 \pm 0.3$ & $8.45 \pm 0.25$ & $6.72 \pm 0.21$ & $5.40 \pm 0.17$ & $3.90 \pm 0.14$ & $2.26 \pm 0.12$ \\
\hline$d N_{c h} / d \eta\left(\kappa_{0}\right)$ & 24.1 & 18.5 & 14.8 & 12.4 & 10.5 & 8.4 & 6.4 & 4.8 & 3.4 & 1.9 \\
\hline$d N_{c h} / d \eta\left(\kappa_{\mathrm{eff}}^{m}\right)$ & 22.1 & 17.2 & 14.0 & 11.8 & 10.1 & 8.1 & 6.1 & 4.7 & 3.4 & 1.9 \\
\hline$d N_{c h} / d \eta\left(\kappa_{\mathrm{eff}}^{s+m}\right)$ & 19.8 & 15.4 & 12.4 & 10.4 & 9.1 & 7.4 & 5.6 & 4.3 & 3.1 & 1.8 \\
\hline
\end{tabular}



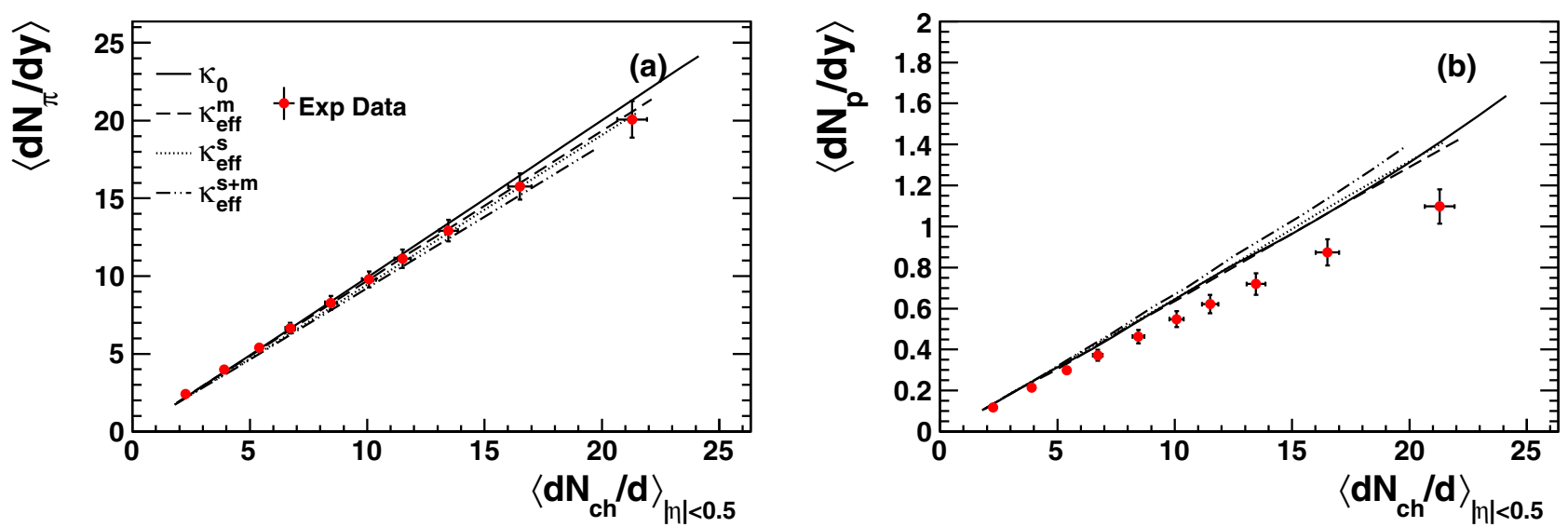

FIG. 4. Pion (a) and proton (b) integrated yields varying with event multiplicity in $p p$ collisions at $\sqrt{s}=7 \mathrm{TeV}$ for different scenarios showing constant string tension setup ( $\kappa_{0}$, solid line), single string-wise tension setup ( $\kappa_{\text {eff }}^{s}$, dotted line), multiple string interaction tension setup $\left(\kappa_{\mathrm{eff}}^{m}\right.$, dashed line), and combined string tension setup $\left(\kappa_{\mathrm{eff}}^{s+m}\right.$, dash-dotted line). The experimental data are taken from [7].

strange sector in a $q \bar{q}$ pair, a diquark-antidiquark pair, and a spin-1 diquark-antidiquark pair at string breakups.

Aside from examining the integrated yield of particles as a function of event multiplicity, it is of more interest to understand the relative production of strange particles. Figure 6 shows the baryon-to-meson production ratio in strange $\left(\Lambda / K_{S}^{0}\right)$ and nonstrange $(p / \pi)$ sectors. In the constant string tension scenario represented by the solid line, we
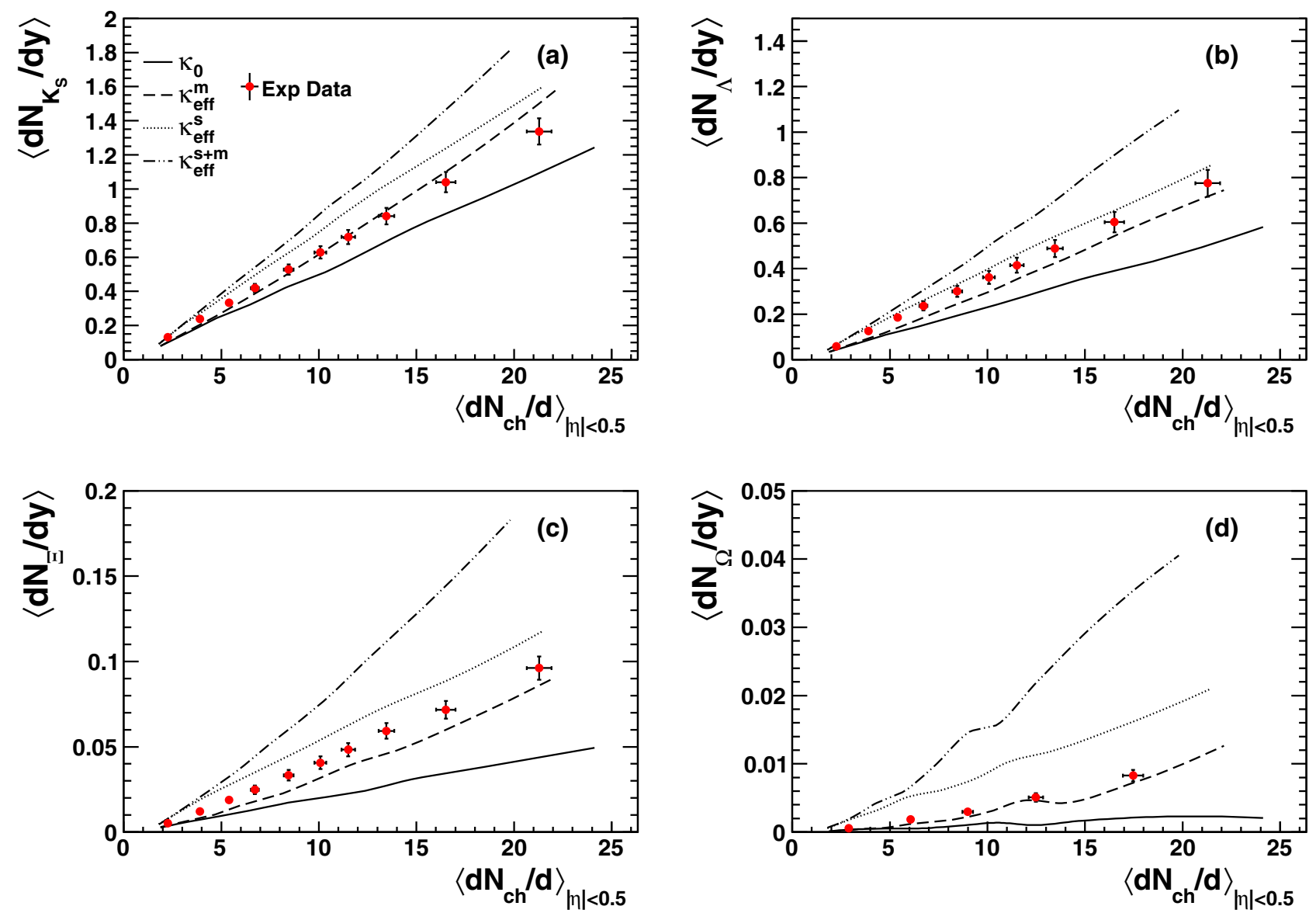

FIG. 5. Strange particle integrated yield varying with event multiplicity in $p p$ collisions at $\sqrt{s}=7 \mathrm{TeV}$ for $K_{S}^{0}$ (a), $\Lambda$ (b), $\Xi$ (c), and $\Omega$ (d). Four different scenarios are shown, with constant string tension setup ( $\kappa_{0}$, solid line), single string-wise tension setup ( $\kappa_{\text {eff }}^{s}$, dotted line), multiple string interaction tension setup ( $\kappa_{\text {eff }}^{m}$, dashed line), and combined string tension setup ( $\kappa_{\text {eff }}^{s+m}$, dash-dotted line). The experimental data are taken from [7]. 

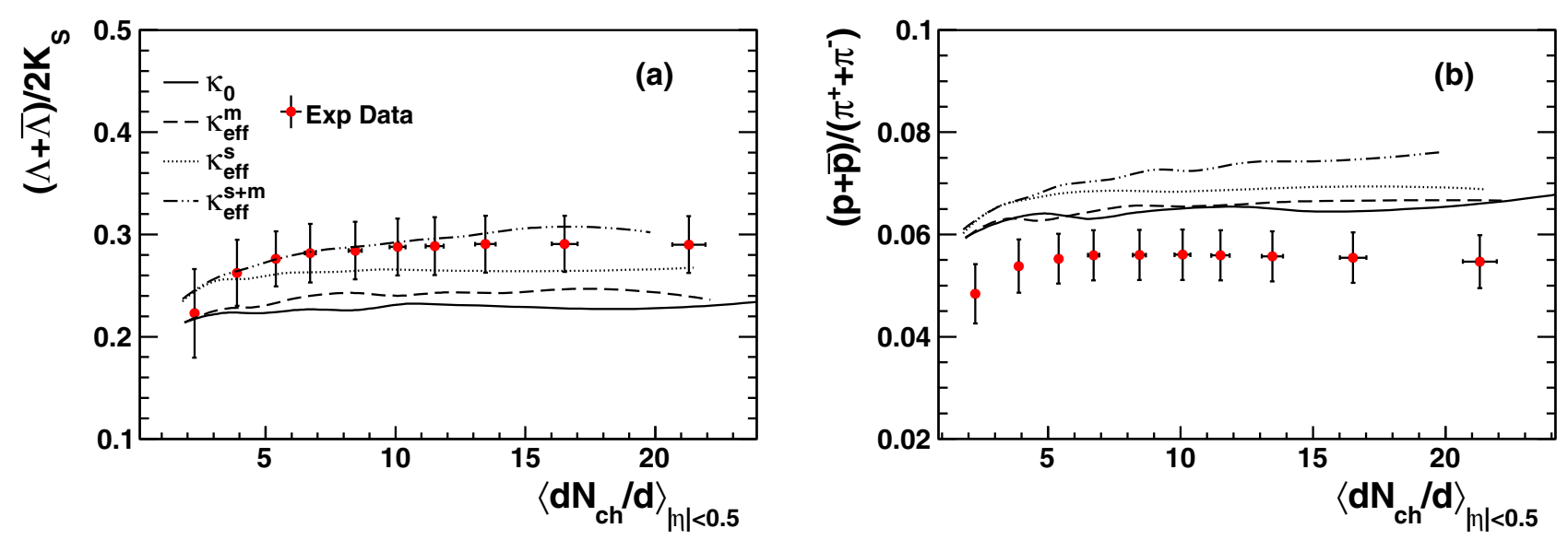

FIG. 6. Baryon-to-meson ratio varying with multiplicity in $p p$ collisions at $\sqrt{s}=7 \mathrm{TeV}$. Four different scenarios are shown, with constant string tension setup $\left(\kappa_{0}\right.$, solid line), single string-wise tension setup $\left(\kappa_{\text {eff }}^{s}\right.$, dotted line), multiple string interaction tension setup $\left(\kappa_{\text {eff }}^{m}\right.$, dashed line). and combined string tension setup ( $\kappa_{\text {eff }}^{s+m}$, dash-dotted line). The experimental data are taken from [7].

observe no dependence of baryon-to-meson ratio on event multiplicity. The inclusion of effective string tension results in a mild increase of both $\Lambda / K_{S}^{0}$ and $p / \pi$ ratios. This is consistent with observations in the experimental data. If we set the $\beta$ parameter to a higher value, the variational string tension assumptions may lead to a stronger increasing trend with event multiplicity.

The strange particle production relative to $\pi$ production is shown in Fig. 7 for $K_{S}^{0}, \Lambda, \Xi$, and $\Omega$. The rising trend with event multiplicity of the strange-particle-to- $\pi$ ratio can be
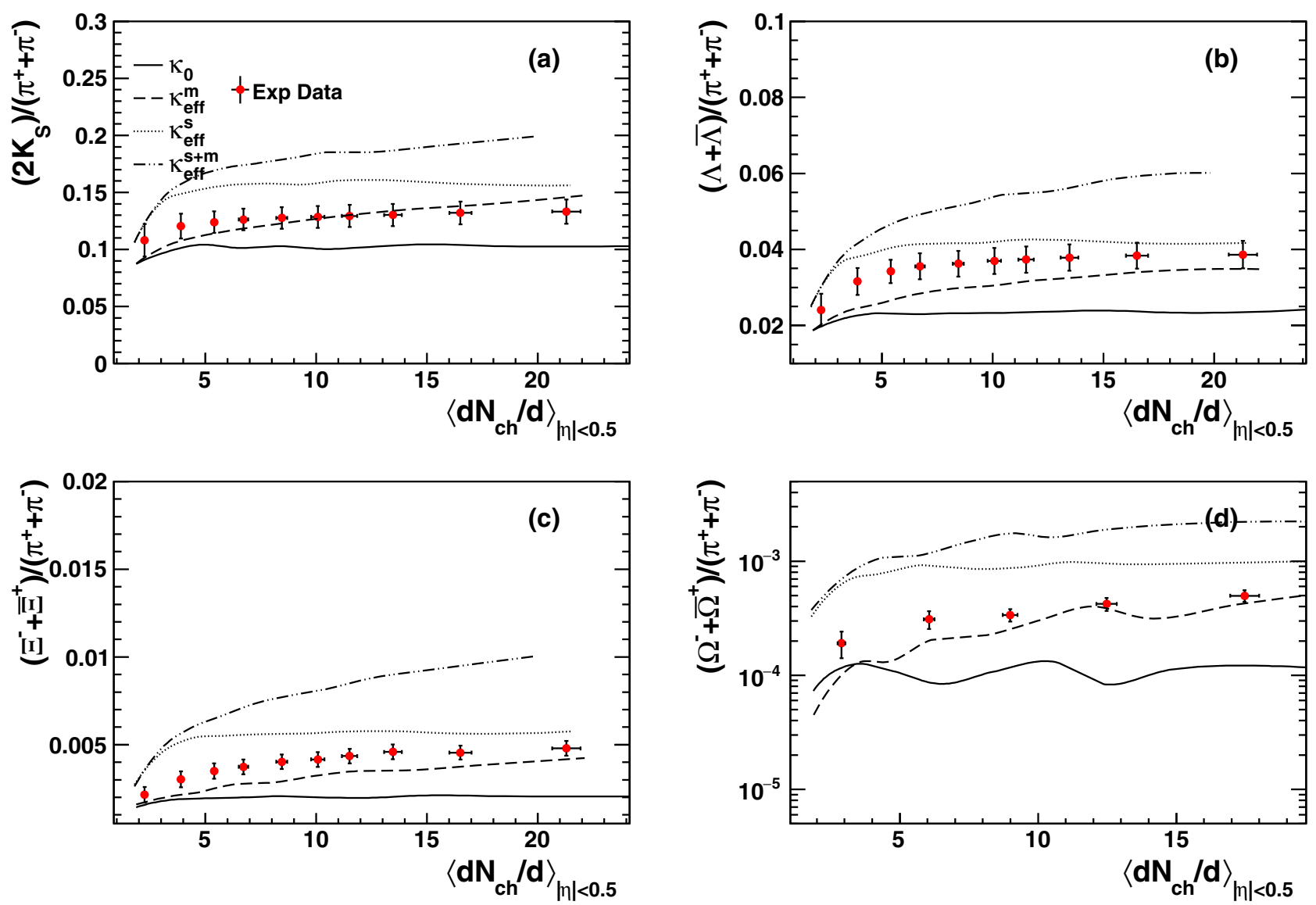

FIG. 7. Strange-particle-to-pion ratio varying with event multiplicity in $p p$ collisions at $\sqrt{s}=7$ TeV. Four different scenarios are shown with constant string tension setup ( $\kappa_{0}$, solid line), single string-wise tension setup ( $\kappa_{\text {eff }}^{s}$, dotted line), multiple string interaction tension setup $\left(\kappa_{\mathrm{eff}}^{m}\right.$, dashed line), and combined string tension setup $\left(\kappa_{\mathrm{eff}}^{s+m}\right.$, dash-dotted line). The experimental data are taken from [7]. 
explained by the inclusion of a string tension variational scheme in the simulation, while a constant string tension assumption gives a flat ratio over the whole event multiplicity range. It is also observed that the gluon wrinkled string structure modification and the multiple string interaction coordinated method implemented in $\kappa_{\text {eff }}^{s}$ and $\kappa_{\text {eff }}^{m}$ predict different event multiplicity dependence of the strange-to-pion ratio. The relative strange production in the $\kappa_{\text {eff }}^{m}$ curve grows monotonically with the event multiplicity, while the $\kappa_{\text {eff }}^{s}$ curve increases dramatically in low multiplicity events and then saturates if the mid-rapidity charged density becomes larger than 4. This observation is consistent with our knowledge of the multiplicity dependent string tension change in two scenarios as described in Fig. 2. The $\kappa_{\text {eff }}^{s+m}$ curve shown with the combined effective string tension change is thus divided into two domains. The rapid increase in the low multiplicity region is dominated by the impact of the gluon wrinkle effect as in $\kappa_{\text {eff }}^{s}$. The interstring interaction takes over and leads to a mild increase of the strange-to-pion ratio in high multiplicity events. Taking account of the strangeness number of the particles analyzed in this comparison, one can also find that the relative production of multistrange particles are more sensitive to the event multiplicity variation than $K_{S}^{0}$ and $\Lambda$. Additionally, one may also find that the effective string tension modification caused by multiple string interaction becomes very small in low multiplicity events. Thus, strange-to0pion ratios at $\left\langle d N_{c h} / d \eta\right\rangle \sim 2$ are grouped into two categories: $\kappa_{\text {eff }}^{m} \approx \kappa_{0}$ and $\kappa_{\text {eff }}^{s+m} \approx \kappa_{\text {eff }}^{s}$. The single string structure relevant effect comes from the gluon radiation inside a string object, thus the impact is going to be stronger with the growth of collision energy [32]. It is therefore natural to see that the modifications introduced by $\kappa_{\mathrm{eff}}^{s}$ or $\kappa_{\mathrm{eff}}^{s+m}$ relative to the $\kappa_{0}$ scenario are still visible even in the low multiplicity events at the LHC energy scale. This multiplicity dependent strangeness enhancement feature can be well understood in the Lund string fragmentation framework by taking the single string structure variation and multiple string interaction mechanisms into consideration.

\section{DISCUSSIONS AND CONCLUSIONS}

The multiplicity dependent strangeness enhancement observed in $p p$ or $p$ - $\mathrm{Pb}$ collisions is an intriguing finding which triggers a lot of theoretical interest and continuing experimental investigations for a wider range of energy dependence. We provide a systematic study of strange particle production in high energy $p p$ collisions based on several variational string tensions in the string fragmentation framework. It turns out that in the $\kappa_{\mathrm{eff}}^{m}$ case, the strange particle yields are generally in agreement with the experimental data. However, the $p / \pi$ ratio is still overestimated with the current parameter setup for all different string tension cases. The multiplicity dependent strangeness enhancement can be naturally included in the string tension change mechanism. The effective string tension change caused by multiple string interactions can offer a reasonable starting point for the observed increasing trend of strange flavor composition, suggesting the multistring interactions are of great interest to explain the multiplicity dependence.

In addition, the effective string tension in Eq. (4), $\kappa_{\mathrm{eff}}^{m}$, can also be extended to other collision types by revising the relevant term to include the nuclear size dependence explicitly. We will leave the comparisons of the extensions to $p-\mathrm{Pb}$ and $\mathrm{Pb}-\mathrm{Pb}$ to the next work. It must be noticed that only the flavor composition effect, which can be directly handled by the tunneling mechanism, is considered in the current framework. Other collective-like effects, such as the near side ridge in two-particle correlations or the radial flow observed in the transverse momentum distribution of high multiplicity $p p$ collisions, still require the inclusion of other mechanisms to deal with the kinematic effect of string dynamics in the high density environment.

In conclusion, this study provides a systematic comparison between theoretical and experimental results of the flavor composition relevant collective behavior in $p p$ collisions based on the string fragmentation scheme. It is a starting point for further exclusive studies combining different mechanisms in a more detailed modeling with microscopic tracing of the parton and hadron evolution history.

\section{ACKNOWLEDGMENTS}

This work was supported by the Fundamental Research Funds for the Central Universities, China University of Geosciences (Wuhan) Grant No. CUG180615, the National Natural Science Foundation of China (11775094, 11475149), the Innovative Research Funds for the Central Universities (2005170503) and the National Key Research and Development Program of China (2016YFE0100900).
[1] J. Rafelski and B. Muller, Phys. Rev. Lett. 48, 1066 (1982); 56, 2334(E) (1986).

[2] P. Koch, B. Muller, and J. Rafelski, Phys. Rep. 142, 167 (1986).

[3] E. Andersen et al., Phys. Lett. B 433, 209 (1998).

[4] J. Adams et al. (STAR Collaboration), Phys. Rev. Lett. 92, 182301 (2004).

[5] B. B. Abelev et al. (ALICE Collaboration), Phys. Lett. B 728, 216 (2014); 734, 409(E) (2014).

[6] J. Rafelski, Eur. Phys. J. A 51, 114 (2015).

[7] J. Adam et al. (ALICE Collaboration), Nat. Phys. 13, 535 (2017).
[8] V. Khachatryan et al. (CMS Collaboration), J. High Energy Phys. 09 (2010) 091.

[9] S. Chatrchyan et al. (CMS Collaboration), Phys. Lett. B 718, 795 (2013).

[10] V. Khachatryan et al. (CMS Collaboration), Phys. Lett. B 765, 193 (2017).

[11] G. Aad et al. (ATLAS Collaboration), Phys. Lett. B 725, 60 (2013).

[12] B. B. Abelev et al. (ALICE Collaboration), Phys. Lett. B 726, 164 (2013).

[13] V. Vislavicius and A. Kalweit, arXiv:1610.03001. 
[14] T. Pierog, I. Karpenko, J. M. Katzy, E. Yatsenko, and K. Werner, Phys. Rev. C 92, 034906 (2015).

[15] J. Aichelin and K. Werner, Phys. Rev. C 79, 064907 (2009); 81, 029902(E) (2010)

[16] T. S. Biro, H. B. Nielsen, and J. Knoll, Nucl. Phys. B 245, 449 (1984).

[17] B. Andersson and P. A. Henning, Nucl. Phys. B 355, 82 (1991).

[18] C. Bierlich, G. Gustafson, L. Lönnblad, and A. Tarasov, J. High Energy Phys. 03 (2015) 148.

[19] T. Sjöstrand, S. Ask, J. R. Christiansen, R. Corke, N. Desai, P. Ilten, S. Mrenna, S. Prestel, C. O. Rasmussen, and P. Z. Skands, Comput. Phys. Commun. 191, 159 (2015).

[20] N. Fischer and T. Sjöstrand, J. High Energy Phys. 01 (2017) 140.

[21] B.-H. Sa, D.-M. Zhou, Y.-L. Yan, X.-M. Li, S.-Q. Feng, B.-G. Dong, and X. Cai, Comput. Phys. Commun. 183, 333 (2012).

[22] B. Andersson, G. Gustafson, G. Ingelman, and T. Sjostrand, Phys. Rep. 97, 31 (1983).
[23] J. Schwinger, Phys. Rev. 82, 664 (1951).

[24] A. Casher, H. Neuberger, and S. Nussinov, Phys. Rev. D 20, 179 (1979).

[25] N. K. Glendenning and T. Matsui, Phys. Rev. D 28, 2890 (1983).

[26] A. Tai and B.-H. Sa, Phys. Lett. B 409, 393 (1997).

[27] T. Sjostrand, S. Mrenna, and P. Z. Skands, J. High Energy Phys. 05 (2006) 026.

[28] B. Andersson, G. Gustafson, and T. Sjostrand, Phys. Scr. 32, 574 (1985).

[29] P. Z. Skands, Phys. Rev. D 82, 074018 (2010).

[30] V. Khachatryan et al. (CMS Collaboration), Phys. Rev. Lett. 105, 022002 (2010).

[31] K. Aamodt et al. (ALICE Collaboration), Eur. Phys. J. C 68, 345 (2010).

[32] H.-Y. Long, S.-Q. Feng, D.-M. Zhou, Y.-L. Yan, H.-L. Ma, and B.-H. Sa, Phys. Rev. C 84, 034905 (2011). 